\title{
Dynamic scaling and temperature effects in thin film roughening
}

\author{
T. A. de Assis \\ Instituto de Física, Universidade Federal da Bahia, Campus Universitário da \\ Federação, Rua Barão de Jeremoabo s/n, 40170-115, Salvador, BA, Brazil \\ Instituto de Física, Universidade Federal Fluminense, Avenida Litorânea s/n, \\ 24210-340 Niterói RJ, Brazil \\ E-mail: thiagoaa@ufba.br
}

\section{F. D. A. Aarão Reis}

Instituto de Física, Universidade Federal Fluminense, Avenida Litorânea s/n, 24210-340 Niterói RJ, Brazil

E-mail: reis@if.uff.br

\begin{abstract}
.
The dynamic scaling of mesoscopically thick films (up to $10^{4}$ atomic layers) grown with the Clarke-Vvedensky model is investigated numerically for broad ranges of values of the diffusion-to-deposition ratio $R$ and lateral neighbor detachment probability $\epsilon$, but with no barrier at step edges. The global roughness scales with the film thickness $t$ as $W \sim t^{\beta} /\left[R^{3 / 2}(\epsilon+a)\right]$, where $\beta \approx 0.2$ is the growth exponent consistent with Villain-Lai-Das Sarma (VLDS) scaling and $a=0.025$. This general dependence on $R$ and $\epsilon$ is inferred from renormalization studies and shows a remarkable effect of the former but a small effect of the latter, for $\epsilon \leq 0.1$. For $R \geq 10^{4}$, very smooth surfaces are always produced. The local roughness shows apparent anomalous scaling for very low temperatures $\left(R \leq 10^{2}\right)$, which is a consequence of large scaling corrections to asymptotic normal scaling. The scaling variable $R^{3 / 2}(\epsilon+a)$ also represents the temperature effects in the scaling of the correlation length and appears in the dynamic scaling relation of the local roughness, which gives dynamic exponent $z \approx 3.3$ also consistent with the VLDS class.
\end{abstract}

PACS numbers: 68.55.-a, 68.35.Ct, 81.15.Aa , 05.40.-a 


\section{Introduction}

Stochastic modeling of molecular-beam epitaxy (MBE) attracted much interest in the last decades due to the importance of this technique to produce high quality thin films for many applications [1. Those models adopt simple rules for the aggregation, diffusion, and desorption processes, consequently allowing the study of morphological properties of large samples [2, 3, 4. In the simplest cases, they assume limited mobility (LM) of adatoms; some examples are the models of Wolf and Villain [5] and of Das Sarma and Tamborenea [6], in which short-range surface diffusion and permanent aggregation take place immediatly after adsorption. More realistic models consider thermally activated microscopic processes and are frequently called colletive diffusion models. The most prominent example is the Clarke-Vvedensky (CV) model [7, in which the adatom diffusion coefficients have Arrhenius forms, with energy barriers depending on the local number of lattice neighbors. An important difference from LM models is that the $\mathrm{CV}$ model obeys detailed balance conditions, thus it may also be a reliable description of the film dynamics without deposition [3, 8].

In the basic formulation of the $\mathrm{CV}$ model, the dynamics may be represented by temperature-like parameters $R$ and $\epsilon$, respectively representing the diffusion-todeposition ratio of isolated atoms in terraces and the detachment probability at step edges [4, 7. For the description of specific MBE processes, at submonolayer or multilayer regime, the models usually include additional energy barriers for diffusion across step edges (upward and downward movements) and additional adatom interactions; a thorough review of homoepitaxy applications is presented in Ref. 4 and recent advance is discussed in Refs. 9, 10, 11, 12. The formation of patterns, growth and coarsening of mounds were some of the features that attracted much interest.

However, a small number of works analyzed the dynamic scaling of surface roughness in CV-type models. This analysis provides a set of scaling exponents connecting the model to stochastic growth equations [2, which helps to distinguish the essential physico-chemical mechanisms of film growth. Initial works on the basic CV model suggested temperature-dependent exponents and anomalous scaling of surface roughness [13, 14, 15, 16. Subsequently, renormalization studies [17, 18, 19] suggested that it belongs to the class of the Villain-Lai-Das Sarma (VLDS) growth equation 20, 21. In $2+1$ dimensions, VLDS scaling was numerically confirmed for $\epsilon=0$ and a broad range of $R$ in Ref. [22] and for some values of $R$ and $\epsilon>0$ in Ref. [23.

The aim of this paper is to perform a systematic investigation of dynamic scaling in the basic CV model (without additional barriers across edges), with particular attention on the role of the temperature-like parameters. First, we study the scaling of global and local surface roughness and confirm that it belongs to the VLDS class for a broad range of values of $R$ and $\epsilon$. Deviations from this scaling are shown to appear only when the surfaces are very flat. Second, we will show evidence of asymptotic normal scaling, but with an apparent anomaly for short times and small $R$ similar to other VLDS models 24]. Finally, we will show that the roughness scales with $R$ in a form similar to the irreversible aggregation model, with a weak dependence on the step detachment rate $\epsilon$. Although this model without barriers at step edges is of limited applicability to real solid films, these results may help the analysis of dynamic scaling in extended versions of the CV model, particularly if crossover features have to be analyzed.

The rest of this paper is organized as follows. In Sec. 2, we present the model 
and the related growth equation. In Sec. 3, we discuss the dynamic scaling of the global surface roughness. In Sec. 4 the scaling of local surface roughness is analyzed. In Sec. [5 we present our conclusions.

\section{Basic definitions and concepts}

\subsection{Model and simulations}

The CV model is defined in a simple cubic lattice, with an initially flat substrate at $z=0$. Deposition occurs with a flux of $F$ atoms per site per unit time, in the $z$ direction towards the substrate. Each adatom occupies one lattice site, whose side is taken as the unit length. We impose the solid-on-solid condition (i. e. overhangs are not allowed), thus only adatoms at the top of each substrate column are mobile.

The hopping rate of an adatom with no lateral neighbor is

$$
D_{0}=\nu_{0} \exp \left(-E_{s} / k_{B} T\right)
$$

where $\nu_{0}$ is a frequency, $E_{s}$ is an activation energy, and $T$ is the temperature. The adatom step occurs in a randomly chosen substrate direction $( \pm x, \pm y)$, towards the top of a NN column. If an adatom has $n$ lateral neighbors, its hopping rate is

$$
D=D_{0} \epsilon^{n} \quad, \quad \epsilon \equiv \exp \left(-E_{b} / k_{B} T\right),
$$

where $E_{b}$ is a bond energy.

An important parameter of the model is the diffusion-to-deposition ratio

$$
R \equiv \frac{D_{0}}{F}=\frac{\nu_{0}}{F} \exp \left(-E_{s} / k_{B} T\right) .
$$

It is usually interpreted as the number of steps of an adatom in a terrace before it is buried by the next atomic layer. However, it is highly probable that an adatom meets a lateral neighbor before being buried, which restricts that interpretation [22]. In the original CV model, $\nu_{0}=2 k_{B} T / h$, where $h$ is the Planck's constant, as predicted by transition state theory [7. Some authors adopted that form 25, but it is more frequent that a constant value $\nu_{0} \sim 10^{12} s^{-1}$ is considered in simulation and analytical works 4. Here we will follow this trend and consider a fixed ratio $\nu_{0} / F=10^{13}$.

Our simulations will be limited to deposition of $10^{4}$ monolayers. It is a typical value for thin films, corresponding to thicknesses of order $2-3 \mu m$, possibly more for molecular materials. The simulation time $t$ will be expressed in number of deposited layers. The substrate size is $L=1024$, which is large enough to avoid finite-size effects in the chosen deposition time. The values of $E_{s}$ and $E_{b}$ are determined by material properties. Thus, since $R=\frac{\nu_{0}}{F} \epsilon^{E_{s} / E_{b}}$, the parameters $R$ and $\epsilon$ simulataneously vary with the temperature for a given material. However, here we are interested in exploring a variety of physico-chemical conditions, which include different values of activation energies. For this reason, $R$ and $\epsilon$ will be taken as the independent parameters of the model.

We will perform simulations in the range $10 \leq R \leq 10^{4}$. Larger values are expected in many MBE processes, but we will show that they produce very smooth surfaces up to the maximal simulated thicknesses. We will also restrict our study to $\epsilon \leq 0.1$, since larger values of this parameter would represent a solid close to the melting point. 


\subsection{Dynamic scaling and universality classes}

The main quantity to characterize the film surface is the local roughness $w(r, t)$ in boxes of size $r$ at time $t$. For calculating this quantity, a square box of lateral size $r$ glides along the film surface and, at each position, the root-mean-square (rms) height fluctuation of columns inside the box is calculated. The average among all box positions and among different configurations of the film at time $t$ is the local roughness. The global roughness $W(t)$ is measured in the full system size $L$, i. e. $W(t)=w(L, t)$. In this work, very large substrates are considered, thus $L$ has negligible effect on $W$.

In systems with normal roughening (in opposition to anomalous roughening [26]), the expected scaling of the local roughness in large substrates is

$$
w(r, t)=r^{\alpha} f\left(\frac{r}{t^{1 / z}}\right),
$$

where $\alpha$ and $z$ are the roughness and dynamic exponents, respectively, and $f$ is a scaling function. For $x \equiv r / t^{1 / z} \ll 1$ (small box sizes), $g(x)$ is constant; for $x \gg 1$

(large box sizes), the local roughness converges to the global one, $W(t)$, which scales as

$$
W \sim t^{\beta}
$$

where $\beta=\alpha / z$ is the growth exponent.

When growth is dominated by surface diffusion, it is expected to be described by a fourth order stochastic equation in the hydrodynamic limit [2]:

$$
\frac{\partial h(\vec{r}, t)}{\partial t}=\nu_{4} \nabla^{4} h+\lambda_{4} \nabla^{2}(\nabla h)^{2}+\eta(\vec{r}, t),
$$

where $h(\vec{r}, t)$ is the height at position $\vec{r}$ and time $t$ in a $d$-dimensional substrate, $\nu_{4}$ and $\lambda_{4}$ are constants and $\eta$ is a Gaussian, nonconservative noise (the contribution of the average external flux is omitted in Eq. 6). The linear version $\left(\lambda_{4}=0\right)$ is the Mullins-Herring ( $\mathrm{MH})$ equation [27, while the nonlinear case is the VLDS equation [20, 21.

For the VLDS class in $2+1$ dimensions, the best estimates of scaling exponents are given by the conserved restricted solid-on-solid models [28, and are very close to one-loop renormalization values [29]: $\alpha \approx 2 / 3, z \approx 10 / 3$, and $\beta \approx 1 / 5$.

\section{Global roughness scaling}

In Fig. 1(a),we show the roughness evolution for $R=10^{2}$ and several values of $\epsilon$. The time scaling gives $\beta \approx 0.20$. As expected, the trend is that $W$ decreases as $\epsilon$ increases, since the detachment from steps of atoms with one or two lateral bonds helps them to move to positions with lower energy, forming more compact configurations. However, the quantitative effect of $\epsilon$ on the roughness is small.

In Fig. 1 (b), we show the roughness evolution for several values of $R$, with $\epsilon=0.05$. In order to estimate the exponent $\beta$, fits of the data are done only for $W \geq 2$, because smaller roughness corresponds to very smooth surfaces. Those fits give $0.18 \leq \beta \leq 0.22$, in good agreement with the VLDS exponent. For $R=10^{4}$, the roughness is very small at all times simulated here, thus deviations appear.

As shown in Ref. 22, for $\epsilon=0, W$ exceeds 2 units only at $t \sim 10^{5}$; for $\epsilon>0$, this occurs for longer times. Thus, for the values $R \geq 10^{4}$ typical of most MBE processes, the film surfaces grown with the basic CV model are very flat. The formation of 

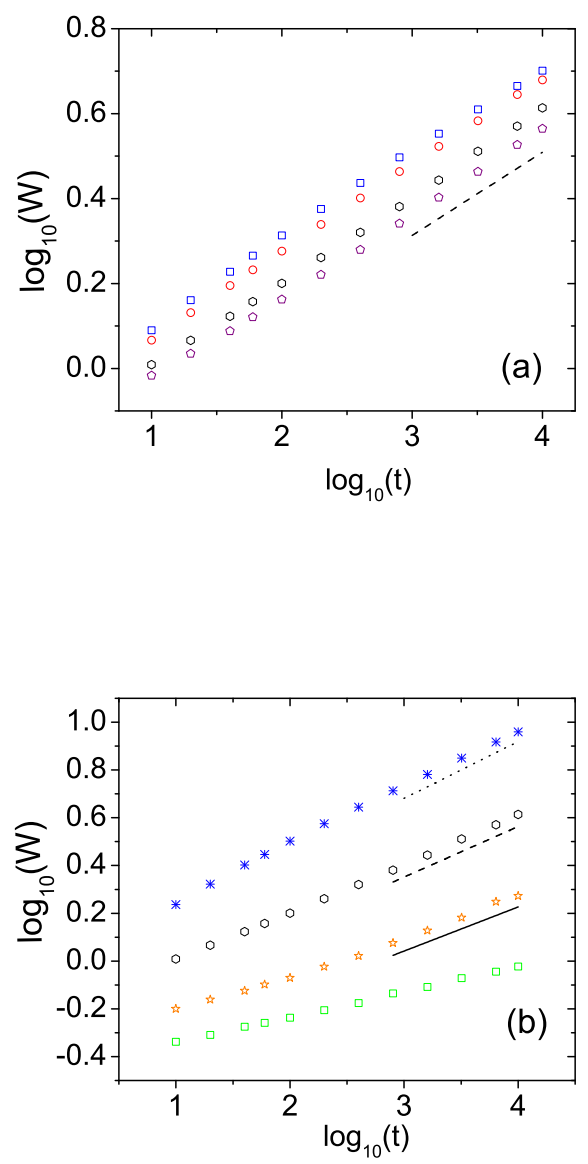

Figure 1. (a) Global roughness as a function of time for $R=10^{2}$, with $\epsilon=0$ (blue squares), 0.01 (red circles), 0.05 (black hexagons), and 0.1 (violet pentagons). The dashed line has slope 0.20. (b) Global roughness as a function of time for $\epsilon=0.05$, with $R=10$ (blue asterisks), $10^{2}$ (black hexagons), $10^{3}$ (orange stars), and $10^{4}$ (green squares). The dotted/dashed/full line has slope 0.22/0.20/0.18.

patterns (e. g. mounds) observed in many simulations is possible only with energy barriers at step edges.

Now we analyze the combined effects of parameters $R$ and $\epsilon$ on the roughness scaling. In the case $\epsilon=0$, Ref. 22] showed that

$$
W \sim \frac{t^{0.2}}{R^{0.3}}
$$

in the growth regime. This was derived from a Family-Vicsek relation [30] that proposed the correlation length as $\xi \sim(R t)^{1 / z}$ [due to the subdiffusive propagation of correlations; $R \propto D$ from Eq. (3)] and the saturation roughness as $W_{s} \sim R^{-1 / 2}$ (due 
to the formation of plateaus of width $R^{1 / z}$ ) [22].

Growth with $\epsilon>0$ allows the detachment of adatoms from steps. It helps filling narrow surface valleys, which reduces the roughness. However, detachment may occur at upward and downward edges of the plateaus, thus is has a small contribution to their size. This explains why $\epsilon>0$ does not lead to drastic changes in the roughness. Consequently, the aggregation of free adatoms at the step edges is still the main mechanism to determine the size of plateaus, and the dependence of $W$ on $R$ is expected to be the same of the model with irreversible step aggregation [Eq. (7)].

The renormalization study of the CV model helps to infer the dependence of the roughness on the detachment probability. Ref. [18] derived the coefficients of the corresponding Langevin equation (with VLDS form) as a function of $D$ and $\gamma \equiv 1-\epsilon$. Those coefficients are products of $D$ or $D \gamma$ by factors of the form $(A+B \epsilon)^{c}$, where $A$ and $B$ are constants that depend on the coefficients of regularization of step functions (which are sensitive to discrete model rules) and $c$ is an integer.

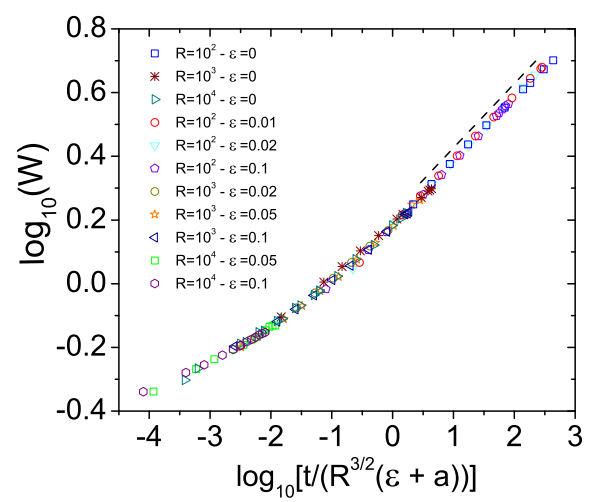

Figure 2. Global roughness as a function of scaled time according to Eq. 87, with $a=0.025$. The dashed line has slope 0.2 .

Following this reasoning, we propose a scaling relation for the $\mathrm{CV}$ roughness as

$$
W \sim \Im\left[\frac{t}{R^{3 / 2}(\epsilon+a)^{\theta}}\right],
$$

where $\Im$ is a scaling function and $a$ and $\theta$ are constants. We did not restrict the scaling to the power-law regime [Eqs. (5) or (7)] because this form may include the initial roughening (small $W$ ).

Fig. 2] shows the roughness for several values of $R$ and $\epsilon$ with time scaled according to Eq. (8). The excellent data collapse with $\theta=1$ and $a=0.025$ confirms the proposed scaling relation. For $\epsilon \lesssim a$, this relation clearly shows the weak dependence of $W$ on that parameter; for $\epsilon<10^{-3}\left(E_{b}>7 k_{B} T\right)$, the effect of the detachment rate is negligible. The scaling of Eq. (8) also includes the case $\epsilon=0$, in which detailed balance fails. 

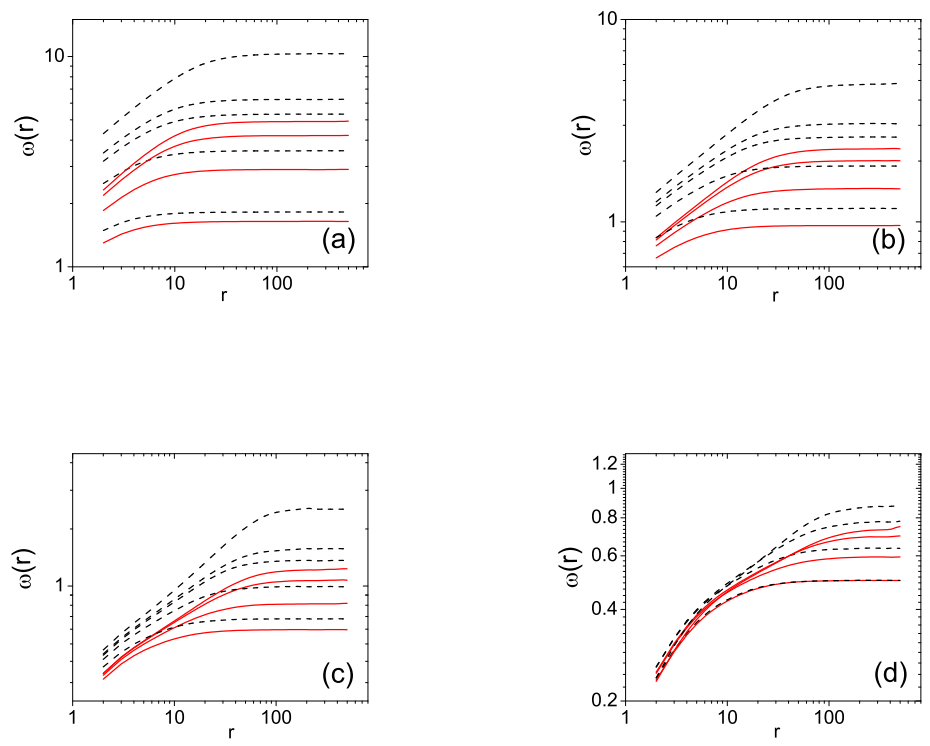

Figure 3. Local roughness as a function of box size for (a) $R=10$, (b) $R=10^{2}$, (c) $R=10^{3}$, and (d) $R=10^{4}$. Dashed (black) and full (red) lines indicate $\epsilon=0.01$ and 0.1 , respectively. For each set of parameters, from bottom to top, deposition times are $t=10,10^{2}, 5 \times 10^{2}$, and $10^{3}$. For $R \leq 10^{3}$ and $\epsilon=0.01$, $t=10^{4}$ is also included .

The effects of $R$ and $\epsilon$ on the roughness are very different from their effects on island size in the submonolayer regime, in which scaling variables of the form $R \epsilon^{m}$ appear in the crossovers (with rational $m>1$ ) 31. Previous works on the basic CV model suggested deviations from VLDS scaling which can be addressed in the light of our results. The results of Figs. 1 a and 1 b are similar to those shown in Ref. [15, which worked in the range $10^{2}<R<10^{4}$. That work suggested the possibility of non-universal exponents due to the small slopes of the fits of large $R$ data, but, as discussed above, roughness scaling is not expected to appear in these conditions. Temperature-dependent exponents $\beta$ were also suggested in Ref. [16], which simulated the model with $R \lesssim 10^{2}$ up to $\approx 10^{3}$ monolayers. A possible crossover from uncorrelated deposition [2] $(\beta=0.5)$ to Edwards-Wilkinson scaling [32] $(\beta=0)$ was suggested. However, the former was obtained for $R \lesssim 1$, in which most atoms cannot execute a single step, and the latter was obtained for very small values of the roughness, before the scaling regime.

\section{Local roughness scaling}

Figs. [3a-d show the local roughness for some values of $R$ and $\epsilon$. The results for small $R$ (100 or less) and small thicknesses (1000 monolayers or less) show features of anomalous roughening, i. e. the $\log w \times \log r$ curves for different times are split for small $r$. This was already observed for $\epsilon=0$ in Ref. 33. 

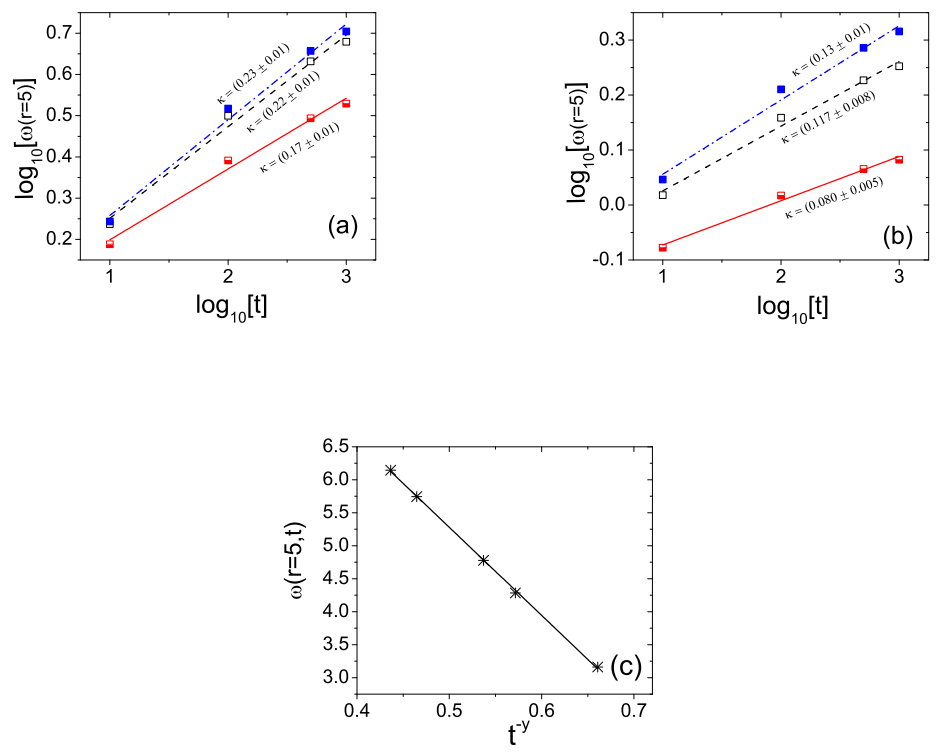

Figure 4. (a), (b): time evolution of the local roughness for fixed box size, $r=5$, respectively for $R=10$ and $R=10^{2}$; detachment parameters are $\epsilon=0$ (blue full squares), $\epsilon=0.01$ (black open squares), and $\epsilon=0.1$ (red half squares); solid lines are linear fits with the effective anomaly exponents shown in the plot. (c) Local roughness at $r=5$ as a function of $t^{-y}$ for $R=10$ and $\epsilon=0.01$, using $y=0.09$.

For $R \geq 10^{3}$, the curves for short times are also split, but coincide after $\approx 100$ monolayers. The values of the local roughness are also very small, thus they would be hard to be distinguished in an experiment. Thus, the anomalous features disappear for typical MBE temperatures $\left(R \geq 10^{5}\right)$; in this case, the presence of anomalous scaling in experimental data is a clear indication of the presence of energy barriers at step edges or other mechanisms that prevent surface smoothing by diffusion.

Figs. 4 (a) and (b) show the time evolution of the roughness for fixed box size, $r=5$, in growth with small $R$ and thicknesses between 10 and 1000. Linear fits of those plots give effective anomaly exponents in the range $0.08 \leq \kappa \leq 0.23$. This procedure parallels the ones used in experimental works. It confirms that thin films grown at very low temperatures may show anomalous scaling features even in the absence of step edge barriers.

However, this anomaly is only apparent, similarly to what occurs in other models in the VLDS class 24]. It is related to slowly vanishing (instead of increasing) terms in the scaling of the local slopes or of the small box local roughness, as

$$
w\left(r_{0}, t\right) \sim A+B t^{-y} .
$$

In Fig. 4 (c), we show $w(5, t)$ versus $t^{-y}$ for $R=10$ and $\epsilon=0.01$, using $y=0.09$. The good linear fit confirms the asymptotically normal scaling, but the small value of $y$ gives large corrections even at long times 24 .

The local roughness scaling does not provide reliable estimates of exponent $\alpha$. 
For small $R$, the scaling region of the $\log w \times \log r$ plot is very short (Fig. 3 3 ) and the approximate slope is much smaller than $\alpha \approx 0.67[28$. For large $R$, a longer scaling region appears, but the slope is also very small. On the other hand, the dynamic exponent $z$ can be estimated by the procedure proposed in Ref. 34]. The first step is to calculate a characteristic length $r_{c}$ which is proportional to the correlation length at a given time $t$. This is obtained by defining $r_{c}$ as

$$
w\left(r_{c}, t\right)=k W(t),
$$

where $W(t)$ is the global width and $k$ is a constant. From Eqs. (5) and (4), it is expected that

$$
r_{c} \sim t^{1 / z}
$$

Here, we consider $k=0.7$ for calculating $r_{c}$.

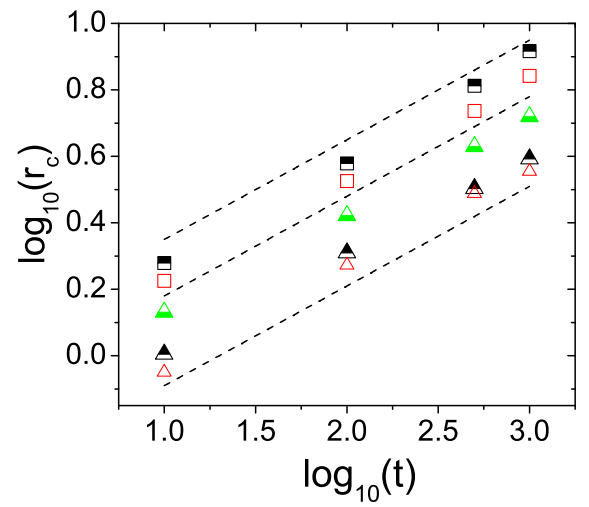

Figure 5. Time evolution of $r_{c}$ for $\mathrm{R}=10$ (triangles) and $\mathrm{R}=100$ (squares). From bottom to top, detachment parameters are $\epsilon=0,0.01,0.1$ for $\mathrm{R}=10$, and $\epsilon=0$, 0.01 for $\mathrm{R}=100$. The dashed lines have slopes 0.3 .

Fig. 5 shows the time evolution of $r_{c}$ for some values of $R$ and $\epsilon$; they are restricted to $R \leq 100$ because the roughness was very small for larger $R$. Linear fits give slopes between 0.30 and 0.32 , which are in excellent agreement with the VLDS value $1 / z \approx 0.30$. It confirms that estimates of dynamic exponents from the local roughness are more reliable than those of roughness exponents [34.

The apparent anomaly for small $R$ does not affect the estimates of $z$ and $\beta$, nor the global roughness scaling shown in Sec. 3. Due to these weak corrections, we propose an scaling relation for the local roughness considering: i) the general form of Family-Vicsek relation (44); ii) the same temperature-dependent variable $R^{3 / 2}(\epsilon+a)$ of the relation (8); iii) the scaling of the correlation length consistent with $\xi \sim(R t)^{1 / z}$ for the case $\epsilon=0$ [22]. This leads to

$$
w(r, t)=\left[\frac{t}{R^{3 / 2}(\epsilon+a)}\right]^{\beta} g\left[\frac{r}{\left(R(\epsilon+a)^{2 / 3} t\right)^{1 / z}}\right],
$$


where $g$ is a scaling function. Fig. 6 shows $w /\left[t /\left(R^{3 / 2}(\epsilon+a)\right)\right]^{\beta}$ as a function of $r /\left[R(\epsilon+a)^{2 / 3} t\right]^{1 / z}$, for several values of $R, \epsilon$, and $t$ (data with very small roughness is excluded, typically for short times or large $R$ ). The data collapse is also good, showing that Eq. (12) contains the leading temperature-dependent terms of the local roughness scaling.

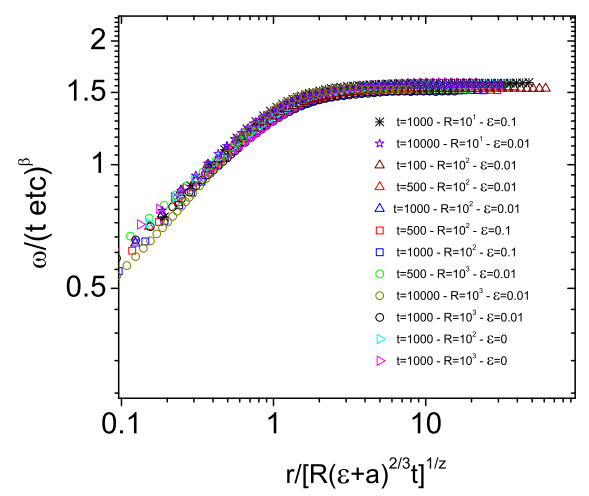

Figure 6. Scaled local roughness as a function of scaled box size.

\section{Conclusion}

We performed simulations of the basic CV model (without extra energy barriers at edges) in $2+1$ dimensions for several values of the diffusion-to-deposition ratio $R$ and detachment probability $\epsilon$ in order to study the scaling of global and local roughness. Relatively short times consistent with thin film growth were considered.

The exponents $\beta(z)$ calculated from the global (local) roughness confirm VLDS scaling with weak corrections. The scaling variable $R^{3 / 2}(\epsilon+a)$, with $a=0.025$, represents the temperature effects in the dynamic scaling relations, with excellent accuracy for the global roughness (time scaling) and small corrections for the local roughness (time and box size scaling). This shows that $R$ is the most important parameter to determine the surface morphology, with much smaller effects of $\epsilon$. This result for thin films is very different from that in submonolayer growth, in which the scaling of island size and related quantities combines rational powers of $R$ and $\epsilon$ [31. On the other hand, the present scaling variables involving $R$ and $\epsilon$ are consistent with results of renormalization studies $[18$.

The local roughness for $R \leq 10^{2}$ shows evidence of anomalous scaling in the range of thicknesses considered here. However, asymptotic normal scaling is observed, with huge corrections expected even at long times. This is consistent with other VLDS models.

Recent works on models with energy barriers for hopping across steps determined exponents $\beta \approx 0.31$ and $1 / z \approx 0.22$ [23, 35] for a range of temperatures; these 
values are quite different from the VLDS exponents. The present model is of limited applicability to real solid films because they usually show this type of additional energy barriers. However, the methods proposed here may be useful for studying those extended models, for instance incorporating the effect of other temperaturedependent variables in the dynamic scaling, or investigating the question of anomalous versus normal roughening.

\section{Acknowledgements}

FDAAR acknowledges support from CNPq and FAPERJ (Brazilian agencies). TAdA also acknowledges CNPq under grant 150874/2014-6. 
Dynamic scaling and temperature effects in thin film roughening

\section{References}

[1] Ohring M, 2001 Materials Science of Thin Films - Deposition and Structure 2nd edn (New York: Academic)

[2] Barabási A L and Stanley H E, 1995 Fractal Concepts in Surface Growth (Cambridge: Cambridge University Press)

[3] Krug J, 1997 Adv. Phys. 46139

[4] Evans J W, Thiel P A, and Bartelt M C, 2006 Surf. Sci. Rep. 611

[5] Wolf D E and Villain J, 1990 Europhysics Lett.13 389

[6] Das Sarma S and Tamborenea P, 1991 Phys. Rev. Lett. 66325

[7] Clarke S and Vvedensky D D, 1988 J. Appl. Phys. 632272

[8] Siegert M and Plischke M, 1992 Phys. Rev. Lett. 682035

[9] Clancy P, 2011 Chem. Mater. 23522

[10] Einax M, Dieterich W, and Maass P, 2013 Rev. Mod. Phys. 85921

[11] Ferrando N, Gosalvez M A and Ayuela A, 2014 J. Phys. Chem. C 11811636

[12] Bommel S et al., 2014 Nat. Commun. 55388

[13] Tamborenea P I and Das Sarma S, 1993 Phys. Rev. E 482575

[14] Das Sarma S, Lanczycki C J, Kotlyar R, and Ghaisas S V, 1996 Phys. Rev. E 53359

[15] Kotrla M and Smilauer P, 1996 Phys. Rev. B 5313777

[16] Meng B and Weinberg W H, 1996 Surf. Sci. 364151

[17] Haselwandter C A and Vvedensky D D, 2007 Europhys. Lett. 7738004

[18] Haselwandter C A and Vvedensky D D, 2008 Phys. Rev. E 77061129

[19] Haselwandter C A and Vvedensky D D, 2008 Int. J. Mod. Phys. B 223721

[20] Villain J, 1991 J. Phys. I 119

[21] Lai Z W and Das Sarma S, 1991 Phys. Rev. Lett. 662348

[22] Aarão Reis F D A A, 2010 Phys. Rev. E 81041605

[23] Leal F F, Ferreira S C, and Ferreira S O, 2011 J. Phys.: Condens. Matter 23292201

[24] Aarão Reis F D A A, 2013 Phys. Rev. E 88022128

[25] Smilauer P and Vvedensky D D, 1995 Phys. Rev. B 5214263

[26] Ramasco J J, López J M, and Rodríguez M A, 2000 Phys. Rev. Lett. 842199

[27] Mullins W W, 1957 J. Appl. Phys. 28 333; Herring C, 1951 The Physics of Powder Metallurgy (W. E. Kingston, McGraw-Hill, New York)

[28] Reis F D A A, 2004 Phys. Rev. E 70031607

[29] Janssen H K, 1997 Phys. Rev. Lett. 781082

[30] Family F and Vicsek T, 1985 J. Phys. A 18 L75

[31] Oliveira T J and Aarão Reis F D A, 2013 Phys. Rev. B 87235430

[32] Edwards S F and Wilkinson D R, 1982 Proc. R. Soc. London Ser. A 38117

[33] de Assis T A and Aarão Reis F D A, 2013 J. Stat. Mech. P10008

[34] Chame A and Reis F D A A, 2004 Surf. Sci. 553145

[35] Leal F F, Oliveira T J, and Ferreira S C, 2011 J. Stat. Mech. P09018 